\title{
Calculated Absorption and Vibrational Circular Dichroism Spectra of Fundamental and Overtone Transitions for a Chiral HCCH Molecular Fragment in the Hypothesis of Coupled Dipoles
}

\author{
ROBERTO GANGEMI, GIOVANNA LONGHI, AND SERGIO ABBATE* \\ Dipartimento di Scienze Biomediche e Biotecnologie, Università di Brescia, Brescia, Italy \\ Dedicated to Prof. Piero Salvadori, Pisa, on the occasion of his 70th birthday.
}

\begin{abstract}
We establish the general behavior of absorption and vibrational circular dichroism spectra (VCD) of a chiral HCCH fragment lacking all symmetry elements; the study is limited to $\mathrm{CH}$-stretching modes, and the Hamiltonian employed is written in terms of normal-mode coordinates and momenta and approximates two different Morse oscillators interacting through a harmonic coupling term; rotational strengths are evaluated within a hypothesis of coupled electric dipoles. Van Vleck contact transformations written in terms of raising and lowering operators are used to calculate spectra up to the manifold $\Delta v=4$. Three transformations are necessary to obtain fourthorder terms in the relevant operators, namely, the electric and magnetic dipole moments. The dynamics of the system exhibits 1:1 resonance terms in addition to the Darling-Dennison coupling term. We discuss the importance of coupling between $\mathrm{CH}$ stretches with respect to differences in their local mechanical characteristics in determining the aspect of the absorption and VCD fundamental and overtone spectra of increasing quantum number. (c) 2005 Wiley-Liss, Inc. Chirality 17:530-539, 2005.
\end{abstract}

KEY WORDS: vibrational circular dichroism (VCD); $\mathrm{CH}$ stretching; overtones; near infrared (NIR); local modes; normal modes; Van Vleck perturbation theory

\section{STATEMENT OF THE PROBLEM AND METHOD}

In collaboration with Professor Salvadori's group, some years ago we measured the vibrational circular dichroism (VCD) spectra in the near-infrared (NIR) range of some limonene-like compounds ${ }^{1}(\Delta v=3,4)$ and of cyclic ketones $^{2}(\Delta v=2,3,4)$. Establishing whether any behavior was common to the various overtone orders and what kinds of relations exist in the fundamental $\mathrm{CH}$-stretching region were important theoretical goals.

In a previous paper, ${ }^{3}$ we tackled this theoretical goal by applying the Van Vleck perturbative method ${ }^{4}$ to the study of the $\mathrm{CH}$-stretching vibrational dynamics of the molecular fragment $\mathrm{HCCH}$ with $C_{2}$ symmetry; specifically, we modeled the fragment as two coupled anharmonic oscillators with the same mechanical frequencies, the same anharmonicities, etc. A wholly algebraic treatment of the problem was made possible by the computer-based algebraic manipulator Maple. ${ }^{5}$ In this way, we could obtain analytical expressions for frequencies $(\omega)$, dipole strengths $(D)$, and rotational strengths $(R)$ for $\mathrm{CH}$ stretching transitions with $\Delta v=1,2,3$. Actually, experimentalists can measure rotational strengths for overtone spectra at least up to $\Delta v=41,2,6-8$ and dipole strengths much further; 9,10 besides, most experimental data involve a variety of molecular fragments that not only (c) 2005 Wiley-Liss, Inc. are dyssymmetric but are quite asymmetric and possess many degrees of freedom. In the present paper, our purpose is to extend our study to the third overtone, $\Delta v=4$, on one hand and, on the other hand, to a fragment that still possesses two degrees of freedom but which is made up of $\mathrm{CH}$ oscillators that have two different mechanical frequencies and/or two different anharmonicities. The motivation of the latter generalization is that we wish to compare with NIR-VCD data of ring molecules, for which it is known that mechanical frequencies, anharmonicity constants, and bond lengths are different for axial and equatorial $\mathrm{CH}$ bonds. ${ }^{10-12}$ Thus, the $\mathrm{HCCH}$ fragment discussed herein might be found in 6- or 5-membered ring molecules, like $(3 R)-(+)$-methylcyclohexanone and $(3 R)$ (+)-methylcyclopentanone, with an axial $\mathrm{CH}$ at one end and an equatorial $\mathrm{CH}$ at the other end.

In Professor Salvadori's laboratory in 1985, two of us (S.A. and G.L.) made the first VCD measurements in the range $1300-800 \mathrm{~nm}$.

${ }^{*}$ Correspondence to: Sergio Abbate, Dipartimento di Scienze Biomediche e Biotecnologie, Università di Brescia, Viale Europa 11, 25123 Brescia, Italy. E-mail: abbate@med.unibs.it

Received for publication 16 March 2005; Accepted 22 June 2005

DOI: $10.1002 /$ chir.20202

Published online 27 September 2005 in Wiley InterScience (www.interscience.wiley.com). 
These generalizations require that few more terms be formally included in the Hamiltonian of Ref. 3, which is written as a fourth-order Taylor expansion in normal coordinates $q_{+}, q_{-}$(vide infra):

$$
H=H_{0}+\varepsilon H_{1}+\varepsilon^{2} H_{2}
$$

The different oscillators $\mathrm{C}_{1} \mathrm{H}_{1}$ and $\mathrm{C}_{2} \mathrm{H}_{2}$, are modeled as Morse oscillators in local coordinates $l_{1}$ and $l_{2}, 13$ with distinct mechanical frequencies $\omega_{01}$ and $\omega_{02}$ and/or distinct anharmonicity constants $\chi_{1}$ and $\chi_{2}$. The two local Morse oscillators are supposed to be linearly coupled via a harmonic interaction force constant, $K_{12} \cdot 3,10,14,15$ We can then write the terms of Eq. (1) as follows:

$$
\begin{aligned}
H_{0}= & \frac{h c}{2}\left[\omega_{+}\left(\frac{p_{+}^{2}}{\hbar^{2}}+q_{+}^{2}\right)+\omega_{-}\left(\frac{p_{-}^{2}}{\hbar^{2}}+q_{-}^{2}\right)\right], \\
\varepsilon H_{1}= & h c\left[K_{+++} q_{+}^{3}+K_{---} q_{-}^{3}+K_{++-} q_{+}^{2} q_{-}\right. \\
& \left.+K_{+--} q_{-}^{2} q_{+}\right], \\
\varepsilon^{2} H_{2}= & h c\left[K_{++++} q_{+}^{4}+K_{---} q_{-}^{4}+K_{++--} q_{+}^{2} q_{-}^{2}\right. \\
& \left.+K_{+++-} q_{+}^{3} q_{-}+K_{+---} q_{-}^{3} q_{+}\right],
\end{aligned}
$$

where $q_{+}, p_{+}, q_{-}$, and $p_{-}$are the dimensionless coordinate and conjugated momentum operators associated with the higher- and lower-frequency normal modes; they reduce respectively to symmetric and antisymmetric combinations of bond-stretching coordinates (or momenta) in the case of identical oscillators and for $K_{12}>0$. The normalmode frequencies are

$$
\omega_{+}^{2}=\frac{\omega_{01}^{2}+\omega_{02}^{2}}{2}+\frac{1}{2} \sqrt{\left(\omega_{01}^{2}-\omega_{02}^{2}\right)^{2}+\frac{K_{12}^{2}}{4 \pi^{4} c^{4} m^{2}}}
$$

and

$$
\omega_{-}^{2}=\frac{\omega_{01}^{2}+\omega_{02}^{2}}{2}+\frac{1}{2} \sqrt{\left(\omega_{01}^{2}-\omega_{02}^{2}\right)^{2}+\frac{K_{12}^{2}}{4 \pi^{4} c^{4} m^{2}}}
$$

In these equations, $m=(12 / 13) \mathrm{amu}$ is the reduced mass of each $\mathrm{CH}$ bond, and $c$ is the speed of light. The constants $K_{+++}$through $K_{+-{ }_{+}}$in Eqs. $\left(2^{\prime \prime}\right)$ and $\left(2^{\prime \prime \prime}\right)$ can be written in terms of $\omega_{01}, \omega_{02}, \chi_{1}$, and $\chi_{2}$ and $K_{12}$, analogously to that done, e.g., in Ref. 3 (see Appendix I). The terms with an odd number of "-" are 0 in the case of identical oscillators and $K_{12}>0$.

The use of Morse oscillators to model local anharmonic oscillators, while not strictly necessary, allows easy definition of the parameters $\omega_{0 i}$ and $\chi_{i} \cdot{ }^{13}$ Indeed, the expansion in Eq. (1) is stopped at fourth order and the use of coupled fourth-order generic anharmonic oscillators in principle would be satisfactory as long as the third-order term in the potential were opposite the second- and fourth-order terms, with relations between expansion coefficients as required by the Morse potential. ${ }^{13-16}$
The Van Vleck method consists of transforming the Hamiltonian to a new representation in which only degenerate (or nearly degenerate) states are coupled. The effective Hamiltonian, $H^{(n)}$, obtained after $n$ Van Vleck transformations, as well as the effective operators $\mu^{(n)}$ and $m^{(n)}$ needed for calculating dipole and rotational strengths, can be obtained ${ }^{3,4}$ by

$$
H^{(n)}=T_{n} \ldots T_{1} H T_{1}^{-1} \ldots T_{n}^{-1}, \quad \text { with } \quad T_{n}=e^{i \varepsilon^{n} S_{n}} .
$$

As pointed out in Ref. 3, fourth-order expansion of the electric and magnetic dipole moments is required to obtain non-zero intensity at $\Delta v=4$, which makes it necessary to perform at least three contact transformations: $T_{1}, T_{2}$, and $T_{3}$. The generating function $S_{1}$ and likewise $S_{2}$ was determined for the simple Hamiltonian of Ref. 3 by requiring the off-diagonal terms of the matrix elements generated by the once (and then twice)-transformed Hamiltonian to be zero. In order to determine the generating function coefficients $^{3}$ by this method, an equal number of independent conditions in the matrix elements must be found. Because finding an entire set of independent equations proves to be pretty difficult, even more so as the order of $S$ increases, we came to consider the approach expounded upon here in and used in a number of instances described by Sibert (see e.g. Ref. 17), in which the raising and lowering operators $a^{\dagger}$ and $a$ are employed instead of $p$ and $q$. The relations for raising and lowering operators $a_{+} \dagger, a_{-} \dagger$ and $a_{+}, a_{-}$to $q_{+}, p_{+}$, and $q_{-}, p_{-}$are as follows:

$$
\begin{array}{ll}
q_{+}=\frac{1}{\sqrt{2}}\left(a_{+}+a_{+}^{\dagger}\right), & \frac{p_{+}}{\hbar}=\frac{i}{\sqrt{2}}\left(a_{+}-a_{+}^{\dagger}\right), \\
q_{-}=\frac{1}{\sqrt{2}}\left(a_{-}+a_{-}^{\dagger}\right), & \frac{p_{-}}{\hbar}=\frac{i}{\sqrt{2}}\left(a_{-}-a_{-}^{\dagger}\right) .
\end{array}
$$

Basically, the advantage of this approach lies in the fact that determination of the $S$ functions is made much easier: the conditions can be imposed directly on operators instead of on matrix elements; the expressions for the perturbative terms as well as for the $S$ function are more compact; and, most importantly, they are formally the same for each successive transformation (vide infra). The convention adopted in order to have a univocal form for the transformed operators is that all $a^{\dagger}$ operators precede the $a$ operators in the single terms of the summation, i.e., the normal form, as defined in Ref. 17, is to be used. This can always be done by exploiting the commutation rule, $\left[a, a^{\dagger}\right]=1$. So, by way of example, one obtains for $H_{1}$ :

$$
\varepsilon H_{1}=\sum_{\substack{\mathbf{m}, \mathbf{n} \\|\mathbf{m}|+|\mathbf{n}| \leq 3}} c_{\mathbf{m}, \mathbf{n}} a^{\dagger \mathbf{m}} a^{\mathbf{n}}
$$

having introduced the arrays of integers $\mathbf{m}=\left\{m_{+}, m_{-}\right\}, \mathbf{n}=$ $\left\{n_{+}, n_{-}\right\}$, with $|\mathbf{m}|+|\mathbf{n}|=m_{+}+m_{-}+n_{+}+n_{-}$, where when we write $a^{\dagger \mathbf{m}} a^{\mathbf{n}}$ we mean $a_{+}^{\dagger \mathrm{m}+} \cdot a_{\underline{1}}^{\lfloor\mathrm{m}}-\cdot a_{+}^{\mathrm{n}+} \cdot a_{-}^{\mathrm{n}-}$. Coefficients $c_{\mathbf{m}, \mathbf{n}}$ are functions of $K_{+++}, K_{++-}$, etc., and are obtained from Eqs. (2"), (4), and $\left(4^{\prime}\right)$ and by reordering the operators. Out of this expression, $S_{1}$ can be easily deduced by a 
choice of all of those addenda, whose denominators are non-zero, through the following equation ${ }^{17}$ :

$$
S_{1}=\sum_{\substack{\mathbf{m}, \mathbf{n} \\|\mathbf{m}|+|\mathbf{n}| \leq 3}}^{\prime \prime} \frac{c_{\mathbf{m}, \mathbf{n}} a^{\dagger \mathbf{m}} a^{\mathbf{n}}}{\mathrm{i} \hbar(\mathbf{m}-\mathbf{n}) \cdot \omega},
$$

where we have introduced $\omega=\left\{\omega_{01}, \omega_{02}\right\}$. The symbol $\Sigma^{\prime \prime}$ means that the sum is over the subset of all terms belonging to $H_{1}$ with $\mathbf{m}, \mathbf{n}$ such that $(\mathbf{m}-\mathbf{n}) \cdot \omega \neq 0 ; \Sigma^{\prime}$ is employed, instead of denoting a sum that is extended over the remaining terms of $H_{1}$, namely, the ones forming the transformed Hamiltonian, $H_{1}^{(1)}$ :

$$
H_{1}^{(1)}=\sum_{\substack{\mathbf{m}, \mathbf{n} \\|\mathbf{m}|+|\mathbf{n}| \leq 3}}^{\prime} c_{\mathbf{m}, \mathbf{n}} a^{\dagger \mathbf{m}} a^{\mathbf{n}} .
$$

The transformed Hamiltonian $H^{(1)}=H_{0}+\varepsilon H_{1}^{(1)}+\varepsilon^{2} H_{2}^{(1)}$ is obtained through the use of Eq. (3). In our case, $H_{1}^{(1)}=0$. The procedure is iterated for $H_{2}^{(1)}$, reduced to "normal form" as in Eq. (5), to build $S_{2}$ and $H_{2}^{(2)}$, which will be given by equations that are formally identical to Eqs. (6) and (7), respectively.

We then generated the final expression for the transformed Hamiltonian $H^{(3)}$, and for $S_{3}$ in $a$ and $a^{\dagger}$ up to third order:

$$
H^{(3)}=H_{0}+\varepsilon H_{1}^{(3)}+\varepsilon^{2} H_{2}^{(3)}+\varepsilon^{3} H_{3}^{(3)}
$$

Complete expressions for $H_{n}^{(2)}$ and $H_{n}^{(3)}$ are too long to report here. In Appendix II, we compare the simple and physically meaningful $H_{0}+H_{2}^{(2)}$ with what has already been reported in the literature ${ }^{18}$ by variational methods. In fact, $H_{0}+H_{2}^{(2)}$ is the usual second-order Hamiltonian in terms of $a$ and $a^{\dagger}$ and is the Darling-Dennison Hamiltonian in the case of identical oscillators. We also point out that, with the two oscillators being different, the terms $q_{+} q_{-}^{3}$ and $q_{+}^{3} q_{-}$of Eq. ( $\left.2^{\prime \prime \prime}\right)$ generate a new 1:1 resonance for $\omega_{+} \approx \omega_{-}$besides the Darling-Dennison 2:2 resonance due to $q_{+}^{2} q_{2}^{2}$. This resonance had already been shown by Lehmann ${ }^{19}$ in studying $\mathrm{HCN}$ and DCN and is also important in determining the absorption spectra of molecules with similar but not identical oscillators; Lehmann himself advised that this resonance in the presence of inequivalent $\mathrm{CH}$ bond stretches be taken into account. An interesting case of this, for example, is that of $n$-paraffins that contain $\mathrm{CH}_{2} \mathrm{~S}$ with slightly different $\mathrm{CH}$ bonds due to missing overall $\sigma$ symmetry generated by conformational disorder, 20 as is the case of cyclic molecules described above.10-12

\section{RESULTS AND DISCUSSION}

Because this work is concerned with the intensity and rotational strength calculations of overtones, we need apply every contact transformation defined by Eq. (3) to the electric and magnetic dipole moments $\mu$ and $\mathbf{m}$, which are usually described in terms of $q$ and $p$ operators. For this reason we have written $S_{1}, S_{2}$, and $S_{3}$ in terms of $q$ and $p$; numerical substitutions of all parameters was needed due to the size of the algebraic expression of $S_{3}$ coeffi- cients. To calculate dipole and rotational strengths, we must first prescribe the functional dependence of electric and magnetic moment operators on coordinates and momenta. A general Taylor expansion of $\boldsymbol{\mu}$ and $\mathbf{m}$ in $q_{ \pm}$and $p_{ \pm}$can be used in principle. However, in order to explicitly define the expansion coefficients and to build corresponding spectra, we have chosen here a bond approximation model for $\boldsymbol{\mu}$ and $\mathbf{m}$ (i.e., we represent molecular dipoles as sums of bond dipoles); further, we have assumed the coupled dipole model for rotational strengths. ${ }^{3,21} \mathrm{We}$ are well aware of the limitations of this model and quite recently, we examined examples wherein the bond approximation for the rotational strengths of overtones proves to be inadequate ${ }^{22}$; however, the simplicity of this model permits us to use a low number of parameters: these are the first and second derivatives of the CH-bond electric dipole moment with respect to the bond-stretching coordinates and they may be evaluated experimentally from overtone intensities. ${ }^{12,14-16}$ In the present case, we use an extension of the coupled-dipoles model presented in Ref. 21, allowing for electrical anharmonicity. The actual values of bond-moment parameters are taken from Ref. 12. Values of the geometric parameters for the $\mathrm{HCCH}$ fragment are reported in the footnote of Table 1 and are the same as in Ref. 3; for dynamic parameters, see below. Transition moments of the transformed operators have to be evaluated with the eigenstates of the transformed Hamiltonian, $H^{(3)}$. Because the matrix associated with $H^{(3)}$ is not diagonal due to $1: 1$ and 2:2 resonances, one proceeds to calculate the eigenvalues (energy levels) and eigenvectors (vibrational modes) on which transition moments have to be evaluated.

As expected from what happens for isolated Morse oscillators, 13 the values of energies for levels through $\Delta v=$ 4 are not modified by third-order terms in $\varepsilon .{ }^{10}$ Regarding dipole and rotational strengths, for $\Delta v=1$ and $\Delta v=3$ they are not affected by the fourth-order terms in $\varepsilon$; on the contrary, at $\Delta v=2$ and $\Delta v=4$, there are corrections in the $\varepsilon^{4}$ terms due to the introduction of $S_{3}$. This can be appreciated by comparing column A of Table 1 relative to the case of identical oscillators with the results of Table IV in Ref. 3. Because we want to illustrate the behavior of the dipole and rotational strengths upon increasing the difference between the oscillators' intrinsic parameters, we report in Table 1 (columns B and E) the output of four parameters' sets for very closely chosen values for local frequencies $\omega_{01}$ and $\omega_{02}$ around $3000 \mathrm{~cm}^{-1}$, anharmonicities $\chi_{1}$ and $\chi_{2}$ around $60 \mathrm{~cm}^{-1}$ and two values for the interaction force constant $K_{12}$ (+0.065 and +0.26 mdyne/ $/ \AA$ ). The first value for $K_{12}$ has been chosen to ensure a separation of $40 \mathrm{~cm}^{-1}$ between the fundamental frequencies in the case $\omega_{01}=\omega_{02}=3000 \mathrm{~cm}^{-1}$ : we observe that the chosen value for $K_{12}$ is not unreasonable as it compares well with experimental values for direct couplings between $\mathrm{CH}$ stretches in $n$-paraffins, as reported in Refs. 23 and 24; the second value instead has been chosen to be four times the first one, in order to compensate for the observed loss of intensity in rotational strengths. The chosen values for $\omega_{01}, \omega_{02}, \chi_{1}, \chi_{2}$ should also be compared with the values obtained, e.g., for axial and equatorial $\mathrm{CH}$ of cyclohexane 


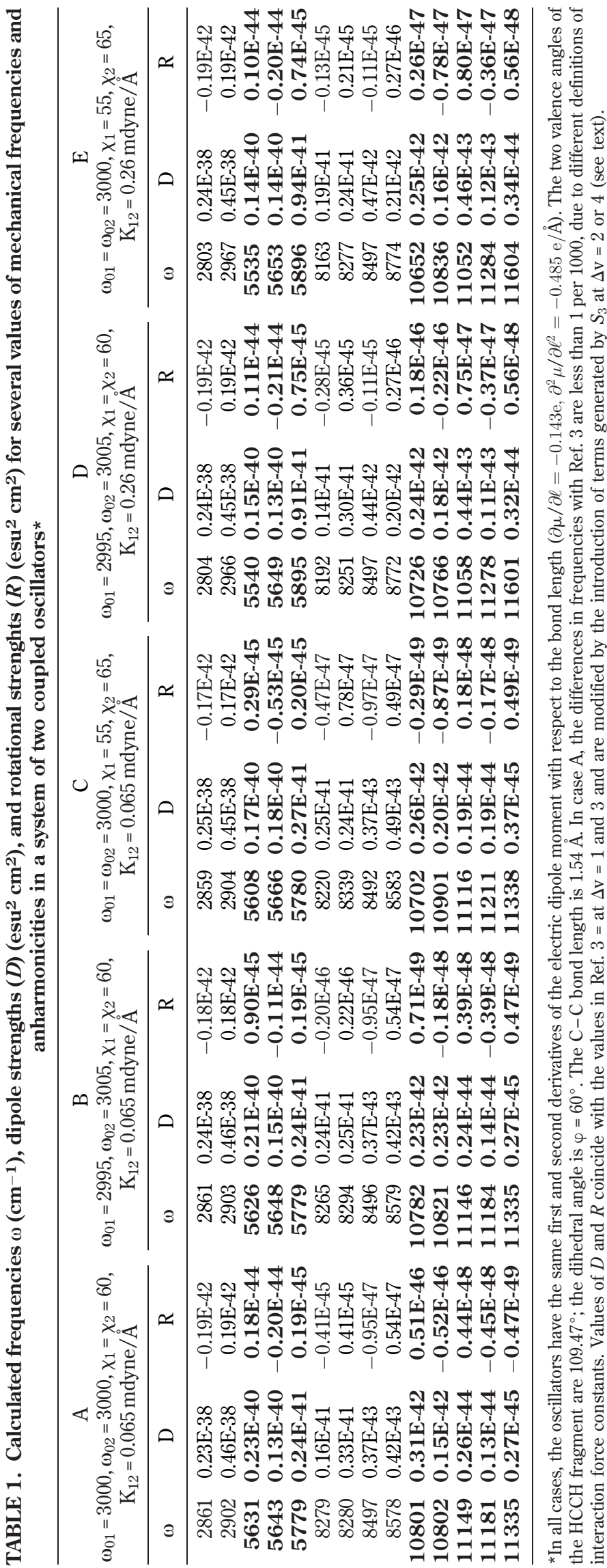


by NIR-local-mode spectroscopy; indeed in Ref. 10: $\omega_{01}=$ $3004 \mathrm{~cm}^{-1}, \omega_{02}=3037 \mathrm{~cm}^{-1}, \chi_{1}=63.1 \mathrm{~cm}^{-1}, \chi_{2}=62.1 \mathrm{~cm}^{-1}$. One may notice that, on increasing the difference between the mechanical frequencies of the $\mathrm{CH}$ bonds $\left(\omega_{0}\right)$ (column B) and between their anharmonicities $(\chi)$ (column $C$ ), the calculated rotational strengths tend to be 1 order of magnitude below those of column A, from the second overtone and upward, signaling that the oscillators can be thought of as uncoupled. If the mechanical frequencies $\omega$ or the anharmonicity constants $\chi$ are too close (column A), then the two lowest-lying features in overtone manifolds, although they exhibit rotational strengths of the correct order of magnitude, ${ }^{1}$ are unobservable, since the two bands of opposite sign tend to cancel out, having almost the same frequencies. In a coupled-oscillator model, to ensure observability of a high overtone VCD for molecules containing an $\mathrm{HCCH}$ fragment, the mechanical characteristics of $\mathrm{CH}$ bonds should be sufficiently similar to favor interaction but not too much, in order to avoid degeneracy. Alternatively, if we increase the coupling constant $K_{12}$ (and leave all other parameters unaltered), the rotational strengths rise again (see columns $\mathrm{D}$ and $\mathrm{E}$ ). Incidentally we notice that, assuming the same value for $\chi_{1}-\chi_{2}$ and for $\omega_{01}-\omega_{02}$, one obtains a greater effect at higher overtone order in the first case, because the effect of anharmonicity goes with the square of vibrational quantum number $v$, while the effect of mechanical frequency is linear in $v$.

The main issues of the present study in relation to the overtone VCD spectra are as follows: (a) Is it possible to associate observed VCD spectra with normal mode states at high overtones $(\Delta v>2)$, and what do the predicted spectra look like? (b) What is the relation between the VCD spectra of a manifold at a given $\Delta v$ with the one at $(\Delta v+1)$ ? For this purpose, it is worthwhile to consider Figure 1, where we report the graphical representation of the results for absorption and VCD spectra corresponding to columns C (top of the Figure) and E (bottom) of Table 1; therein we have prescribed a Lorentzian bandshape to each predicted transition, following the indications of Ref. 25. One may appreciate from the second line of the figure that VCD spectra corresponding to case $\mathrm{C}$ of Table 1 for two coupled oscillators are practically unobservable beyond $\Delta v=2$; indeed, at $\Delta v=3$ and 4 , the order of magnitude of predicted VCD features is too little to make the calculated features observable. This is ultimately due to the small value of $K_{12}$ causing too little coupling between oscillators. With the assumption of coupled-dipole mechanism in generating rotational strengths, the conditions to expect observability of VCD are a greater value for the interaction force constant in addition to a sufficient difference in the
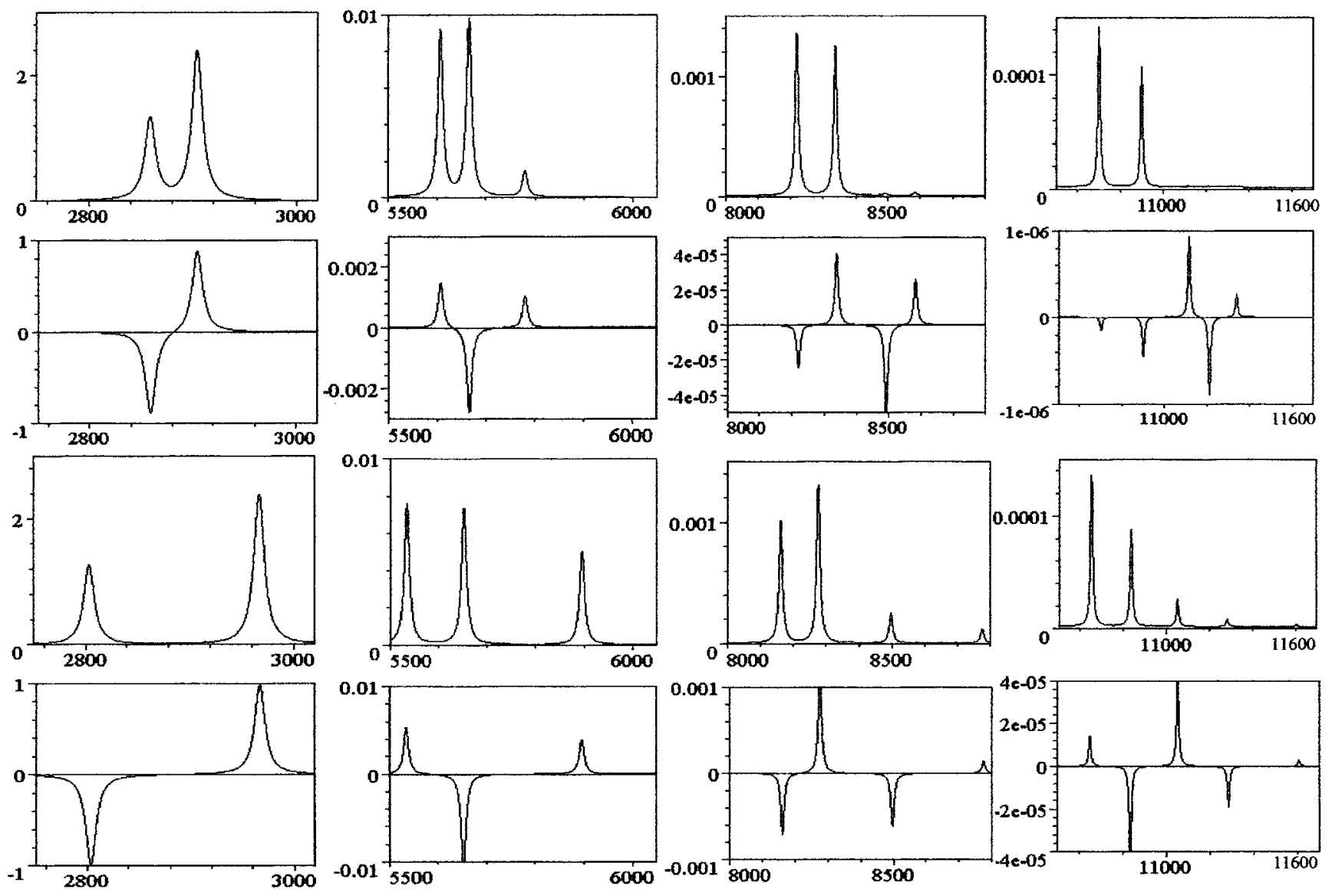

Fig. 1. Calculated absorptions and VCD spectra for the set C (first pair of rows) and set $\mathrm{E}$ (second pair) of Table 1 with $K_{12}>0$. Abscissae are in wavenumber units; band areas are the calculated dipole strengths given in units of $10^{-40}(\mathrm{esu} \mathrm{cm})^{2}$, and the calculated rotational strengths in units of $10^{-44}(\mathrm{esu} \mathrm{cm})^{2}$. The bandshape is Lorentzian with a constant half-width at half-height of $6 \mathrm{~cm}^{-1}$ for all overtone orders. 
mechanical frequencies and/or anharmonicities of the two bonds. In comparing the results for cases $\mathrm{C}$ and $\mathrm{E}$ of Table 1 and Figure 1, one sees that the absorption features-in both cases-and the VCD features (just in case E) decrease by 2 orders of magnitude in going from the fundamental $(\Delta v=1)$ to the first overtone region $(\Delta v=2)$ and by 1 order of magnitude for each successive overtone region. This corresponds to experiment.1,2,6-8 Besides, the separation of calculated VCD features at $\Delta v=3$ (ca. $100 \mathrm{~cm}^{-1}$ ) is in line with current experimental resolution $7,8,22$ : in the case of camphor we observed bands separated ca. $100 \mathrm{~cm}^{-1}$, in the case of $\alpha$-pinene ca. $60 \mathrm{~cm}^{-1}$. Examination of Figure 1 allows one to appreciate further characteristics of the spectroscopic behavior of chirally disposed coupled oscillators at the fundamental and overtone orders. The following observations can be made on these numerical examples: (i) at $\Delta v=2$ and in many cases for $\Delta v=3$, the two largest VCD features are associated with the two lowest-frequency features, that are usually described as due to the two local modes of the two $\mathrm{CH}$ bonds; these bands have some VCD, inasmuch as they preserve some dynamical coupling (cf. Ref. 3); (ii) from $\Delta v=4$, this is no longer true, even though the main absorption features are still those for the overtone "local" modes; (iii) the VCD doublet associated with the two lowest-frequency features alternates in sign going from manifold $v$ to manifold $v+1$; this is due to the positive sign of $K_{12}$. We report in Figure 2 the cases with the same parameters as for cases $\mathrm{C}$ and $\mathrm{E}$ of Figure 1, except that the $K_{12}$ parameter is negative. One may notice that for negative $K_{12}$ the lowest-frequency doublet preserves the sign with increasing $v$. This fact had been already pointed out on a semiclassical basis 26 ; (iv) at $\Delta v=2$ the feature at highest frequency, which is generally thought of as the $(1,1)$ combination band in the local-mode quantum numbers, is predicted to be quite strong also in absorption when $K_{12}=0.26$ mdyne $/ \AA$, and this may have something to do with the puzzlingly intense $(1,1)$ combination bands observed in polyethylene and in $n$-paraffins. ${ }^{20}$

To explain sign alternation between successive manifolds in the case of positive $K_{12}$ (point iii above), one may notice that the character of the mode associated with the lowest-frequency feature alternates, i.e., at $\Delta v=1$ it is mainly (in terms of local mode quantum numbers) $\left|1,0>_{1}-\right| 0,1>_{1}$, at $\Delta v=2$ it is mainly $\left|2,0>_{1}+\right| 0,2>_{1}$, at $\Delta v=3$ it is mainly $\left|3,0>_{1}-\right| 0,3>_{1}$, and so forth; for the second-lowestfrequency feature, at $\Delta v=1$ we checked that it is mainly $\left|1,0>_{1}+\right| 0,1>_{1}$, at $\Delta v=2$ it is mainly $\left|2,0>_{1}-\right| 0,2>_{1}$, at $\Delta v=3$ it is mainly $\left|3,0>_{1}+\right| 0,3>_{1}$, and so on. The transformation from normal mode basis to local mode basis can be achieved following the procedure illustrated at the end of Section II of Ref. 3 . The fact that the mostly antisymmetric mode has the lowest frequency at $\Delta v=1$ for $K_{12}>0$ and the mostly symmetric one has the lowest frequency at $\Delta v=1$ for $K_{12}<$ 0 is established by the equation preceding Eq. (3) in the
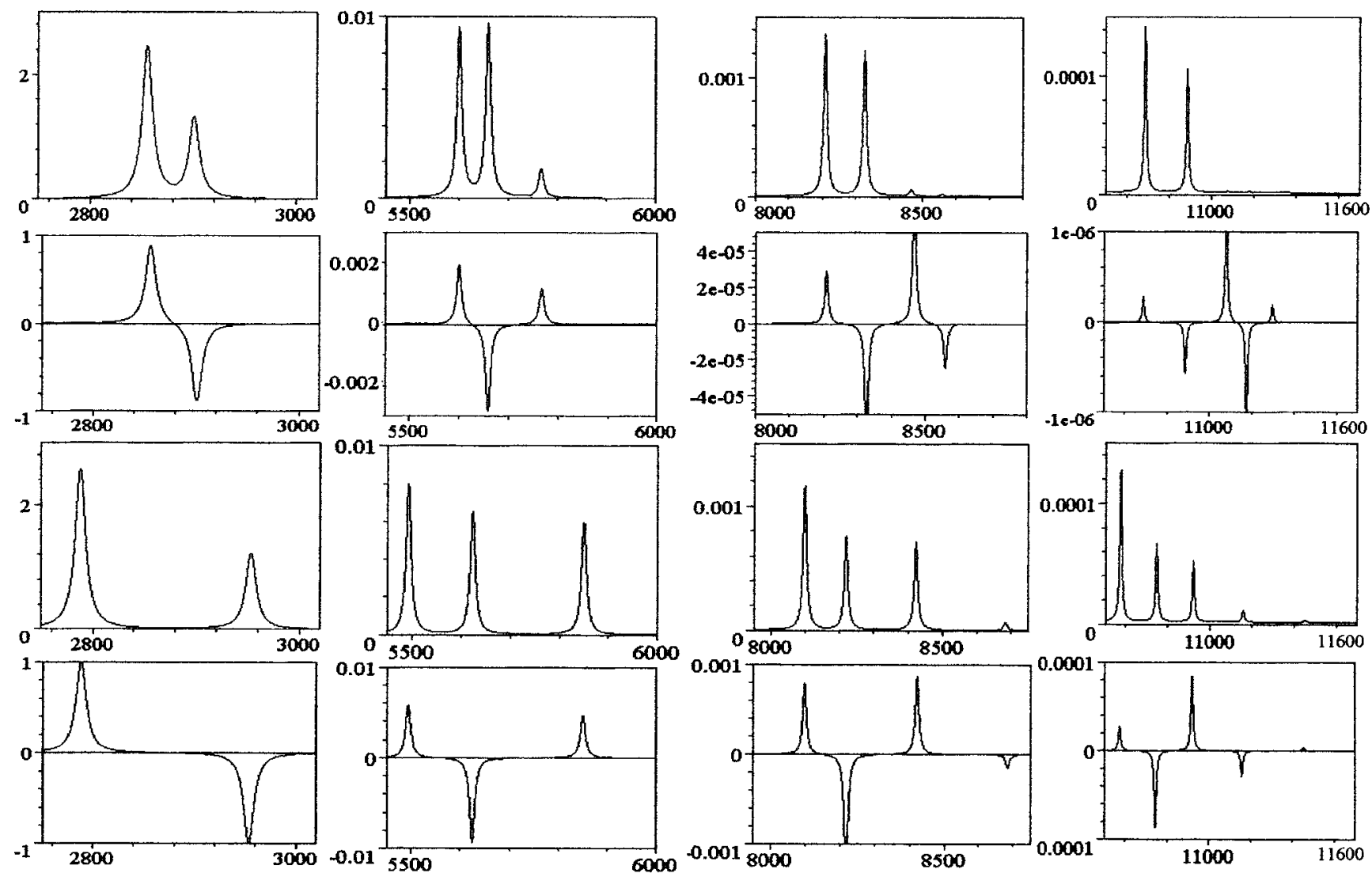

Fig. 2. Calculated absorptions and VCD spectra for set C (first pair of rows) and set $\mathrm{E}$ (second pair) of Table 1 with $K_{12}<0$. Abscissae are in wavenumber units; band areas are the calculated dipole strengths given in units of $10^{-40}(\mathrm{esu} \mathrm{cm})^{2}$, and the calculated rotational strengths in units of $10^{-44}(\mathrm{esu} \mathrm{cm})^{2}$. The bandshape is Lorentzian with a constant half-width at half-height of $6 \mathrm{~cm}^{-1}$ for all overtone orders. 
text and by the results of Appendix I. Indeed, before the Darling-Dennison resonance takes place, the frequency order of $\left|0, v>_{1} \pm\right| v, 0>_{1}$ at each manifold $v$ is the same as $\left|0,1>_{1} \pm\right| 1,0>_{1}$, but that resonance, taking place for submanifolds of separate symmetries, reorders the states; this is true for positive $K_{12}$ values typical of $\mathrm{CH}$-stretching oscillators. The reordering is such that at even values of $v$ the symmetric combination $\left|0, v>_{1}+\right| v, 0>_{1}$ is at the lowest frequency, at odd values of $v$ the lowest-frequency feature is the antisymmetric one $\left|0, v>_{1}-\right| v, 0>_{1}$. This is due to the fact that at even orders the symmetric block of the manifold contains one more state than the antisymmetric one, namely the $\mid v / 2, v / 2_{1}>$ state. This state "pushes" the symmetric local mode combination to low frequencies. This rule ceases to be obeyed at higher overtones due to higher congestion of interacting states. ${ }^{3}$ As noticed above, the sign alternation is not present with $K_{12}<0$ since the lowest-lying feature is the symmetric combination before and after Darling-Dennison resonance: indeed we verified that for all manifolds the lowest-frequency feature is the symmetric one, as is expected, and its VCD is always positive with the present geometry.

The eigenvectors written in terms of local mode coordinates allow one also to appreciate why major absorption features and major VCD features do not necessarily coincide (observation ii above). In the case of different oscillators we have verified that within each manifold the two lowest-frequency features have local mode character, that is to say the contribution in local mode eigenvectors is either from local mode $\mid v, 0>_{1}$ or from $\mid 0, v>1$, while at higher frequencies the contribution of $\mid v, 0>_{1}$ or $\mid 0, v>_{1}$ (these are the modes bringing in intensity in the present bond approximation) is more evenly distributed, even if it is low. This is the reason why in each overtone region not necessarily observed VCD bands correspond to the most intense absorption bands. To be more specific, let us denote by $c_{1 v}$ and $c_{2 v}$ the coefficients multiplying $|v, 0\rangle_{1}$ and $|0, v\rangle_{1}$ respectively, for a given wavefunction $\psi$ at a given overtone order $v$. Within the bond dipole moment hypothesis, one may show that the dipole strength $D_{\psi}$, the rotational strength $R_{\psi}$, and the $g$ factor for the transition from the ground state to $\psi$ are:

$$
\begin{gathered}
D_{\psi}=\mu_{\mathrm{v}}^{2}\left[c_{1 \mathrm{v}}^{2}+c_{2 \mathrm{v}}^{2}+2 c_{1 \mathrm{v}} c_{2 \mathrm{v}} \mathbf{e}_{1} \cdot \mathbf{e}_{2}\right], \\
R_{\psi}=\pi \omega_{\mathrm{v}} \mu_{\mathrm{v}}^{2} c_{1 \mathrm{v}} c_{2 \mathrm{v}} \mathbf{e}_{1} \cdot \mathbf{r}_{\mathbf{C} 1 \mathbf{C} 2} \times \mathbf{e}_{2}, \\
g=4 R_{\psi} / D_{\psi}
\end{gathered}
$$

where $\mathbf{e}_{\mathbf{1}}$ and $\mathbf{e}_{2}$ are the unit vectors of $\mathrm{C}_{1} \mathrm{H}_{1}$ and $\mathrm{C}_{2} \mathrm{H}_{2}$, $\mathbf{r}_{\mathbf{C 1 C 2}}$ is the oriented distance vector from $\mathrm{C}_{1}$ to $\mathrm{C}_{2}$, and $\omega_{v}$ is the wavenumber of the considered transition. We have defined $\mu_{v}=(\partial \mu / \partial r)<0|r| v>+\left(\partial^{2} \mu / \partial r^{2}\right)<0\left|r^{2}\right| v>$ for both $\mathrm{C}_{1} \mathrm{H}_{1}$ and $\mathrm{C}_{2} \mathrm{H}_{2}$. Let us now consider the quantities $R^{\star}=$ $R_{\psi} /\left(\mu_{v}^{2}\right)$ and $D^{*}=D_{\psi} /\left(\mu_{v}^{2}\right)$, which remove the contribution from $\mu_{v}^{2}$ that varies from overtone to overtone. Let us consider case $\mathrm{C}$ of Table 1 and let us see whether these values allow one to interpret the results of Figure 1. From Ref. 3, we have $\mathbf{e}_{\mathbf{1}} \cdot \mathbf{e}_{\mathbf{2}}=0.3333$ and $\mathbf{e} \mathbf{1} \cdot \mathbf{r}_{\mathbf{C} \mathbf{1} \mathbf{2} 2} \times \mathbf{e}_{\mathbf{2}}=1.1855$ A. Thus: for $\Delta v=1$

$\begin{array}{ll}\text { at } 2859 \mathrm{~cm}^{-1} & \text { at } 2904 \mathrm{~cm}^{-1} \\ c_{1}=0.52 & c_{1}=0.85 \\ c_{2}=-0.85 & c_{2}=0.52 \\ R^{\star}=-0.47 \cdot 10^{-4} & R^{\star}=0.48 \cdot 10^{-4} \\ D^{\star}=0.70 & D^{\star}=1.29 \\ g=-2.7 \cdot 10^{-4} & g=1.49 \cdot 10^{-4}\end{array}$

for $\Delta v=2$

at $5608 \mathrm{~cm}^{-1}$

$c_{1}=0.08$

$c_{2}=0.98$

$R^{\star}=0.17 \cdot 10^{-4}$

$D^{\star}=1.01$

$g=0.68 \cdot 10^{-4}$

for $\Delta v=3$

at $8220 \mathrm{~cm}^{-1}$

$c_{1}=0.01$

$c_{2}=-0.99$

$R^{\star}=-0.02 \cdot 10^{-4}$

$D^{\star}=0.97$

$g=-0.09 \cdot 10^{-4}$

for $\Delta v=4$

at $10,702 \mathrm{~cm}^{-1}$

$c_{1}=0.001$

$c_{2}=-1.00$

$R^{\star}=-0.01 \cdot 10^{-4}$

$D^{\star}=1.00$

$g=-0.02 \cdot 10^{-4}$

$$
\begin{aligned}
& \text { at } 5666 \mathrm{~cm}^{-1} \\
& c_{1}=0.96 \\
& c_{2}=-0.12 \\
& R^{\star}=-0.25 \cdot 10^{-4} \\
& D^{\star}=0.87 \\
& g=-1.17 \cdot 10^{-4}
\end{aligned}
$$

$$
\begin{aligned}
& \text { at } 8339 \mathrm{~cm}^{-1} \\
& c_{1}=0.98 \\
& c_{2}=0.02 \\
& R^{\star}=0.05 \cdot 10^{-4} \\
& D^{\star}=0.98 \\
& g=0.19 \cdot 10^{-4}
\end{aligned}
$$

$$
\begin{aligned}
& \text { at } 10,901 \mathrm{~cm}^{-1} \\
& c_{1}=0.99 \\
& c_{2}=-0.0001 \\
& R^{\star}=-0.0003 \cdot 10^{-4} \\
& D^{\star}=0.98 \\
& g=-0.001 \cdot 10^{-4}
\end{aligned}
$$

$$
\begin{aligned}
& \text { at } 5780 \mathrm{~cm}^{-1} \\
& c_{1}=0.17 \\
& c_{2}=0.26 \\
& R^{\star}=0.09 \cdot 10^{-4} \\
& D^{\star}=0.13 \\
& g=3.00 \cdot 10^{-4}
\end{aligned}
$$

$$
\begin{array}{ll}
\text { at } 8492 \mathrm{~cm}^{-1} & \text { at } 8583 \mathrm{~cm}^{-1} \\
c_{1}=0.12 & c_{1}=0.13 \\
c_{2}=-0.11 & c_{2}=0.05 \\
R^{\star}=-0.04 \cdot 10^{-4} & R^{\star}=0.02 \cdot 10^{-4} \\
D^{\star}=0.02 & D^{\star}=0.02 \\
g=-9.49 \cdot 10^{-4} & g=3.66 \cdot 10^{-4}
\end{array}
$$

$$
\begin{aligned}
& \text { at } 11,116 \mathrm{~cm}^{-1} \\
& c_{1}=0.02 \\
& c_{2}=0.10 \\
& R^{\star}=0.01 \cdot 10^{-4} \\
& D^{\star}=0.01 \\
& g=3.48 \cdot 10^{-4}
\end{aligned}
$$

at $11,338 \mathrm{~cm}^{-1}$

$c_{1}=0.05$

$c_{2}=0.015$

$R^{\star}=0.003 \cdot 10^{-4}$

$D^{\star}=0.003$

$g=4.10 \cdot 10^{-4}$ 
This simplified evaluation of $R^{\star}, D^{\star}$, and $g$ gives results in good agreement with those presented in Figure 1 and Table 1, and thus we may understand that the absorption spectrum is dominated by the first two transitions in each overtone manifold $v$, while the VCD spectrum has contribution at higher frequencies; the latter features may be detectable since they have favorable $g$ factor (ca. $10^{-4}$ ), unlike e.g. the first two lowest-frequency features at $\Delta v=4$. However, their detection may be difficult due to the low absorption.

\section{CONCLUSIONS}

In this work we have established what is the general aspect of absorption and VCD spectra for fundamental $(\Delta v=1)$ and overtone $\mathrm{CH}$-stretching transitions up to $\Delta v=$ 4 for a general chiral $\mathrm{HCCH}$ fragment in the case of coupled dipole mechanism. In particular, for positive harmonic interaction constants $K_{12}$ between the two $\mathrm{CH}$ stretching vibrations a scheme of alternating signs in the predicted VCD spectra at successive $\Delta v$ values is expected, whereas a constant scheme is expected at successive $\Delta v$ values for negative $K_{12}$. We have also rationalized why the most intense absorption features do not necessarily coincide with the most intense VCD features. All of this can appreciated by looking at Figures 1 and 2, which are derived for a particular choice of parameters' values; however the method is altogether general and the parameters can be changed if necessary.

We wish to make another couple of comments: we believe that a simple coupled-oscillator model of electric bond dipoles cannot be the only mechanism for generating VCD spectra associated with overtone transitions, unless an enhancement of effective interactions between bonds is present. An important contribution in generating magnetic dipole moments can be given by polarizable groups ${ }^{27}$ or ring currents 28 ; for fundamental transitions such contributions and all others are encompassed by the general theory of vibrational circular dichroism by Stephens ${ }^{29}$ : these mechanisms may even explain high rotational strengths in case of local modes as found in Ref. 22. In any case we think that the first task in understanding overtone VCD spectra is the solution of the anharmonic dynamical problem with special attention to the eigenstates representing high-energy vibrations, and we believe that the present work sheds light onto this aspect.

Last but not least, we observe that the global aspect of Figures 1 and 2 could be arrived at also by a more standard variational approach (see, e.g., Ref. 18). Yet, we think that the Van Vleck-based approach, that has been applied a few times in papers regarding $\mathrm{VCD}, 11,30,31$ can find an easier implementation in packages allowing calculations of general properties, as done already for a particular molecular case by Bak et al. ${ }^{32}$ to treat anharmonicity in the standard IR region. For the $\mathrm{CH}$-stretching overtone region the variant of Van Vleck contact transformation method in terms of raising and lowering operators, that has been developed by Sibert 17 to interpret the absorption spectra and has been extended here to treat VCD, is thought to be necessary to make the problem computationally tractable.

\section{ACKNOWLEDGMENTS}

Financial support is acknowledged from MIUR (Italian Ministry of Education, University, and Research). We thank the reviewers for advice.

\section{APPENDIX I}

We report the coefficients of the studied Hamiltonian obtained from the mechanical parameters of two harmonically coupled Morse oscillators.

$$
\begin{aligned}
& \mathrm{K}_{+++}=-\frac{1}{\sqrt{2}} \frac{\left(\omega_{01}^{2} \sqrt{\chi_{1}} \cos ^{3} \beta+\omega_{02}^{2} \sqrt{\chi_{2}} \sin ^{3} \beta\right)}{\omega_{+}^{3 / 2}} \\
& \mathrm{~K}_{---}=-\frac{1}{\sqrt{2}} \frac{\left(-\omega_{01}^{2} \sqrt{\chi_{1}} \sin ^{3} \beta+\omega_{02}^{2} \sqrt{\chi_{2}} \cos ^{3} \beta\right)}{\omega_{-}^{3 / 2}}, \\
& K_{++-}=-\frac{3}{\sqrt{2}} \frac{\left(-\omega_{01}^{2} \sqrt{\chi_{1}} \cos \beta+\omega_{02}^{2} \sqrt{\chi_{2}} \sin \beta\right) \sin \beta \cdot \cos \beta}{\sqrt{\omega}_{-} \omega_{+}}, \\
& K_{+--}=-\frac{3}{\sqrt{2}} \frac{\left(\omega_{01}^{2} \sqrt{\chi_{1}} \sin \beta+\omega_{02}^{2} \sqrt{\chi_{2}} \cos \beta\right) \sin \beta \cdot \cos \beta}{{\sqrt{\omega_{+}} \omega_{-}}}, \\
& \mathrm{K}_{++++}=\frac{7}{12} \frac{\left(\omega_{01}^{2} \chi_{1} \cos ^{4} \beta+\omega_{02}^{2} \chi_{2} \sin ^{4} \beta\right)}{\omega_{+}^{2}}, \\
& \mathrm{~K}_{----}=\frac{7}{12} \frac{\left(\omega_{01}^{2} \chi_{1} \sin ^{4} \beta+\omega_{02}^{2} \chi_{2} \cos ^{4} \beta\right)}{\omega_{-}{ }^{2}} \\
& \mathrm{~K}_{++--}=\frac{7}{2} \frac{\left(\omega_{01}^{2} \chi_{1}+\omega_{02}^{2} \chi_{2}\right) \sin ^{2} \beta \cos ^{2} \beta}{\omega_{+} \omega_{-}}, \\
& \mathrm{K}_{+++-}=\frac{7}{3} \frac{\left(-\omega_{01}^{2} \chi_{1} \cos ^{2} \beta+\omega_{02}^{2} \chi_{2} \sin ^{2} \beta\right) \sin \beta \cdot \cos \beta}{\sqrt{\omega_{-} \omega_{+}^{3 / 2}}}, \\
& \mathrm{~K}_{+---}=\frac{7}{3} \frac{\left(-\omega_{01}^{2} \chi_{1} \sin ^{2} \beta+\omega_{02}^{2} \chi_{2} \cos ^{2} \beta\right) \sin \beta \cdot \cos \beta}{\sqrt{\omega}_{+} \omega_{-}^{3 / 2}},
\end{aligned}
$$

where $\beta$ is given by:

$$
\beta=\frac{1}{2} \cos ^{-1}\left(\frac{\omega_{01}^{2}-\omega_{02}^{2}}{\sqrt{\left(\omega_{01}^{2}-\omega_{02}^{2}\right)^{2}+4 K_{12}^{2} /\left(4 \pi^{2} c^{2} m\right)^{2}}}\right) .
$$

$\beta$ defines the normal modes as follows:

$$
\begin{aligned}
\Delta l_{1} & =\frac{q_{+} \cos \beta-q_{-} \sin \beta}{\sqrt{m}}, \\
\Delta l_{2} & =\frac{q_{+} \cos \beta+q_{-} \sin \beta}{\sqrt{m}}
\end{aligned}
$$

\section{APPENDIX II}

Here we wish to compare the twice transformed Hamiltonian $H^{(2)}=H_{0}+H_{2}^{(2)}$ with the analogous dynamical 


$$
x_{13}=\frac{2 \mathrm{~K}_{+--}^{2} \omega_{+} \omega_{-}-3 \mathrm{~K}_{+--} \mathrm{K}_{+++} \omega_{+}^{2}+12 \mathrm{~K}_{+--} \mathrm{K}_{+++} \omega_{--}^{2}+\mathrm{K}_{++--} \omega_{+}^{3}-4 \mathrm{~K}_{++--} \omega_{+} \omega_{-}^{2}}{\omega_{+}\left(\omega_{+}^{2}-4 \omega_{-}^{2}\right)},
$$

approximations proposed in the literature ${ }^{18}$ in terms of the raising and lowering operators. We follow the same notation of Ref. 18, i.e., \#1 for the symmetric and \#3 for the antisymmetric normal mode. The correspondence with our notation is:

$$
\mathrm{A}_{1}=a_{+} ; \mathrm{A}_{1}^{+}=a_{+}^{\dagger} ; \mathrm{A}_{3}=a_{-} ; \mathrm{A}_{3}^{+}=a_{-}^{\dagger}
$$

We thus obtained

$$
\begin{aligned}
\frac{H}{h c}= & \omega_{1}\left(\mathrm{~A}_{1}^{\dagger} \mathrm{A}_{1}+\frac{1}{2}\right)+\omega_{3}\left(\mathrm{~A}_{3}^{\dagger} \mathrm{A}_{3}+\frac{1}{2}\right) \\
& +\mathrm{x}_{11}\left(\mathrm{~A}_{1}^{\dagger} \mathrm{A}_{1}\left(\mathrm{~A}_{1}^{\dagger} \mathrm{A}_{1}+1\right)+\frac{1}{4}\right)+\mathrm{x}_{33}\left(\mathrm { A } _ { 3 } ^ { \dagger } \mathrm { A } _ { 3 } \left(\mathrm{~A}_{3}^{\dagger} \mathrm{A}_{3}\right.\right. \\
& \left.+1)+\frac{1}{4}\right)+\mathrm{x}_{13}\left(\mathrm{~A}_{1}^{\dagger} \mathrm{A}_{1} \mathrm{~A}_{3}^{\dagger} \mathrm{A}_{3}+\frac{1}{2}\left(\mathrm{~A}_{1}^{\dagger} \mathrm{A}_{1}+\mathrm{A}_{3}^{\dagger} \mathrm{A}_{3}\right.\right. \\
& \left.+\frac{1}{2}\right)+\frac{1}{4} K_{1133}\left(\mathrm{~A}_{1}^{\dagger} \mathrm{A}_{1}^{\dagger} \mathrm{A}_{3} \mathrm{~A}_{3}+\mathrm{A}_{3}^{\dagger} \mathrm{A}_{3}^{\dagger} \mathrm{A}_{1} \mathrm{~A}_{1}\right) \mathrm{T} \\
& +K_{1113}\left(\mathrm{~A}_{1}^{\dagger} \mathrm{A}_{3}\left(1+\mathrm{N}_{1}\right)+\left(\mathrm{N}_{1}+1\right) \mathrm{A}_{3}^{\dagger} \mathrm{A}_{1}\right) \\
& +K_{1333}\left(\mathrm{~A}_{3}^{\dagger} \mathrm{A}_{1}\left(1+\mathrm{N}_{3}\right)+\left(\mathrm{N}_{3}+1\right) \mathrm{A}_{1}^{\dagger} \mathrm{A}_{3}\right),
\end{aligned}
$$

where we have also introduced the number operators $N_{1}$ and $N_{3}$ for the symmetric and antisymmetric states, namely:

$$
\mathrm{N}_{1}=\mathrm{A}_{1}^{\dagger} \mathrm{A}_{1} \quad \text { and } \quad \mathrm{N}_{3}=\mathrm{A}_{3}^{\dagger} \mathrm{A}_{3}
$$

Apart from the constant term $T$ (see below) and the last two terms (with constants $K_{1113}$ and $K_{1333}$ ), typical of different oscillators, Hamiltonian (A1) formally coincides with that of Halonen. ${ }^{18}$ The constant term $T$ adds to the zero-point energy a term equal to

$$
-\frac{3}{8} K_{++++}+\frac{7}{16} \frac{K_{+++}^{2}}{\omega_{+}}-\frac{3}{8} K_{--}+\frac{3}{16} \frac{K_{++}^{2} \omega_{+}}{\left(\omega_{+}^{2}-4 \omega_{-}^{2}\right)}
$$

which, assuming for the $K$ coefficients a relationship typical of the Morse oscillator (and when the coupling constant vanishes), approaches $-(1 / 4) \times$ (see Ref. 13).

All matrix elements generated by Eq. (A1) coincide with the ones given by Lehmann ${ }^{19}$ in a study of $\mathrm{HCN}$ and $\mathrm{DCN}$.

We report here for completeness all of the constants of Eq. (A1):

$$
\begin{gathered}
x_{11}=\frac{3}{4} \frac{-5 K_{+++}{ }^{2}+2 K_{++++} \omega_{+}}{\omega_{+}}, \\
x_{33}=-\frac{1}{4} \frac{3 K_{+--}^{2} \omega_{+}^{2}-8 K_{+--}^{2}-\omega_{-}^{2}-6 K_{----} \omega_{+}^{3}+24 K_{----} \omega_{+} \omega_{-}^{2}}{\omega_{+}\left(\omega_{+}^{2}-4 \omega_{-}^{2}\right)},
\end{gathered}
$$

$$
\begin{aligned}
K_{1133}= & -\frac{2 K_{+--}^{2} \omega_{-}\left(\omega_{+}+2 \omega_{-}\right)}{\omega_{+}\left(\omega_{+}^{2}-4 \omega_{-}^{2}\right)}-\frac{K_{+--} K_{+++}\left(\omega_{+}^{2}+2 \omega_{-}^{2}\right)}{\omega_{+}\left(\omega_{+}^{2}-4 \omega_{-}^{2}\right)} \\
& +K_{++-},
\end{aligned}
$$$$
K_{1113}=-\frac{1}{4} \frac{\left(29 \omega^{2}+\omega_{--}-5 \omega^{3}+6 \omega^{3}+K_{++-} K_{+++}\right.}{\omega_{+} \omega_{-}\left(4 \omega^{2}{ }_{+}-\omega^{2}+\right)}-\frac{1}{4}
$$$$
\times \frac{\left(15 \omega_{+} \omega_{-}^{4}-8 \omega^{2} \omega_{--}^{3}-47 \omega^{3}{ }_{+}^{2} \omega_{-}^{2}+8 \omega_{-}^{5}+2 \omega_{-}^{5}\right) \mathrm{K}_{+--} \mathrm{K}_{++-}}{\omega_{+} \omega_{-}\left(4 \omega^{2}{ }_{+}-\omega_{-}^{2}\right)\left(\omega^{2}{ }_{+}-4 \omega_{-}^{2}\right)}
$$$$
+\frac{3}{4} \mathrm{~K}_{+++-}
$$$$
K_{1333}=-\frac{1}{4} \frac{\left(29 \omega_{-}^{2} \omega_{+}+6 \omega_{-}^{3}-5 \omega_{+}^{3}\right) \mathrm{K}_{---} \mathrm{K}_{+--}}{\omega_{+} \omega_{-}\left(4 \omega_{-}^{2}-\omega_{+}^{2}\right)}-\frac{1}{4}
$$$$
\times \frac{\left(15 \omega_{+} \omega_{-}^{4}-8 \omega_{+}^{2}+\omega_{-}^{3}-47 \omega_{+}^{3} \omega_{-}^{2}+8 \omega_{+}^{5}+2 \omega_{-}^{5}\right) K_{+--} K_{++-}}{\omega_{+} \omega_{-}\left(4 \omega_{+}^{2}-\omega_{-}^{2}\right)\left(\omega_{+}^{2}-4 \omega_{-}^{2}\right)}
$$$$
+\frac{3}{4} \mathrm{~K}_{+---} \cdot
$$

Expressions (A2) hold for different oscillators; if they are alike, then Eqs. (A2) take up the form given by Halonen.18 The last two terms of Eq. (A1) with the K1113 and K1333 coefficients are related to the 1:1 resonance, as can be easily seen remembering the intrinsic property of the operators, which is to create and to destroy (vibrational) quanta in the oscillator they refer to. The presence of terms containing the number operator implies an amplification of the 1:1 resonance effect with increasing overtone order.

\section{LITERATURE CITED}

1. Abbate S, Longhi G, Ricard L, Bertucci C, Rosini C, Salvadori P, Moscowitz A. Vibrational circular dichroism as a criterion for local mode versus normal mode behaviour. Near infrared circular dichroism spectra of some monoterpenes. J Am Chem Soc 1989;111: 836-840.

2. Abbate S, Longhi G, Boiadjiev S, Lightner DA, Bertucci C, Salvadori P. Analysis of vibrational circular dichroism data in the near infrared and visible range. Enantiomer 1998;3:337-347.

3. Abbate S, Gangemi R, Longhi G. Dipole and rotational strengths for overtone transitions of a $C_{2}$-symmetry $\mathrm{HCCH}$ molecular fragment using Van Vleck perturbation theory. J Chem Phys 2002;117: $7575-7586$.

4. Amat G, Nielsen HH, Tarrago G. Rotation-vibration of polyatomic molecules. New York: Dekker; 1971.

5. MAPLE V. Maplesoft, Inc.: Waterloo, Ontario, Canada; 1998.

6. Keiderling TA, Stephens PJ. Vibrational circular dichroism of overtone and combination bands. Chem Phys Lett 1976;41:46-49.

7. (a) Castiglioni E, Lebon F, Longhi G, Abbate S. Vibrational circular dichroism in the near infrared: instrumental developments and 
application. Enantiomer 2002;7:161-170. (b) Abbate S, Longhi G, Givens III JW, Boiadjiev SE, Lightner DA, Moscowitz A. Observation of vibrational circular dichroism for overtones transitions with commercially available CD-spectrometers. Appl Spectrosc 1996;50: 642-643.

8. (a) Nafie LA, Dukor RK, Roy R, Rilling A, Cao X, Buijs H. Observation of Fourier transform near infrared vibrational circular dichroism to $6150 \mathrm{~cm}^{-1}$. Appl Spectrosc 2003;57:1245-1249. (b) Cao X, Shah RD, Dukor RK, Guo C, Freedman TB, Nafie LA. Extension of Fourier transform vibrational circular dichroism into the near-infrared region; continuous spectral coverage from 800 to $10000 \mathrm{~cm}^{-1}$. Appl Spectrosc 2004;58:1057-1064.

9. Reddy KV, Heller DF, Berry MJ. Highly vibrationally excited benzene: overtone spectroscopy and intramolecular dynamics of $\mathrm{C}_{6} \mathrm{H}_{6}, \mathrm{C}_{6} \mathrm{D}_{6}$, and partially deuterated or substituted benzenes. J Chem Phys 1982; 76:2814-2837.

10. Kjaergaard HG, Henry BR. The relative intensity contributions of axial and equatorial $\mathrm{CH}$ bonds in the local mode overtone spectra of cyclohexane. J Chem Phys 1992;96:4841-4851.

11. Abbate S, Longhi G, Santina C. Theoretical and experimental studies for the interpretation of vibrational circular dichroism in the $\mathrm{CH}$ stretching overtone region. Chirality 2000;12:180-190.

12. Longhi G, Ricard L, Abbate S, Zerbi G. Electric dipole moment functions from vibrational absorption intensities of fundamental and overtone transitions. J Chem Phys 1988;88:6733-6741.

13. Herzberg G. Molecular spectra and molecular structure. I. Spectra of diatomic molecules, 2nd edition. New York: Van Nostrand; 1950.

14. Henry BR. The local mode model and overtone spectra: a probe of molecular structure and conformation. Acc Chem Res 1977;10: 429-435.

15. Child MS, Halonen L. Overtone frequencies and intensities in the local mode picture. Adv Chem Phys 1985;LVII:1-58.

16. Sage ML, Jortner J. Bond modes. Adv Chem Phys 1981;XLVII: $293-323$.

17. Sibert III EL. Theoretical studies of vibrationally excited polyatomic molecules using canonical Van Vleck perturbation theory. J Chem Phys 1988;88:4378-4390.

18. Halonen L. Local mode vibrations in polyatomic molecules. Adv Chem Phys 2000;104:41-178.

19. Lehmann KK. Beyond the $x-K$ Relations. Calculation of $1-1$ and $2-2$ resonance constants with applications to $\mathrm{HCN}$ and DCN. Mol Phys 1989;66:1129-1137.
20. Ricard-Lespade L, Longhi G, Abbate S. The first overtone of CHstretching in polyatomic chains: a conformationally dependent spectrum. Chem Phys 1990;142:245-259.

21. (a) Escribano JR, Freedman TB, Nafie LA. A Bond-origin-independent formulation of the bond dipole model of vibrational circular dichroism. J Phys Chem 1987;91:46-49. (b) Abbate S, Laux L, Overend J, Moscowitz A. A charge flow model for vibrational rotational strengths. J Chem Phys 1981;75:3161-3164.

22. Longhi G, Gangemi R, Lebon F, Castiglioni E, Abbate S, Pultz VM, Lightner DA. A comparative study of overtone $\mathrm{CH}$-stretching vibrational circular dichroism spectra of fenchone and camphor. J Phys Chem A 2004;108:5338-5352.

23. Ricard L, Abbate S, Zerbi G. Conformationally dependent Fermi resonances and long-range interactions between $\sigma$ bonds in polymethylene systems derived from their Raman spectra. J Phys Chem 1985; 89:4793-4799.

24. Snyder RG, Strauss HL, Elliger CA. Carbon-hydrogen stretching mode and the structure of $n$-alkyl chains. Long disordered chains. J Phys Chem 1982;86:5145-5150.

25. Heller DF, Mukamel S. Theory of vibrational overtone line shapes of polyatomic molecules. J Chem Phys 1979;70:463-472.

26. Abbate S, Longhi G, Kwon K, Moscowitz A. The use of crosscorrelation functions in the analysis of circular dichroism spectra. J Chem Phys 1998;108:50-62.

27. (a) Barnett CJ, Drake AF, Kuroda R, Mason SF. A dynamic polarization model for vibrational optical activity and the infrared circular dichroism of a dihydro[5]helicene. Mol Phys 1980;41:455-468. (b) Abbate S, Havel HA, Laux L, Pultz V, Moscowitz A. Vibrational circular dichroism in deuterated phenylethanes. J Phys Chem 1988;92: 3302-3311.

28. Nafie LA, Freedman TB. Ring current mechanisms of vibrational circular dichroism. J Phys Chem 1986;90:763-767.

29. Stephens PJ. Theory of vibrational circular dichroism. J Phys Chem 1985;89:748-752.

30. Faulkner TR, Marcott CA, Moscowitz A, Overend J. Anharmonic effects in vibrational circular dichroism. J Am Chem Soc 1977;99: 8160-8168.

31. Polavarapu P. Vibrational optical activity of anharmonic oscillator. Mol Phys 1996;89:1503-1510.

32. Bak KL, Bludsky O, Jørgensen P. Ab initio calculations of anharmonic vibrational circular dichroism intensities of trans-2,3-dideuteriooxirane. J Chem Phys 1995;103:10548-10555. 